\title{
Deuterated rhodamines for protein labelling in nanoscopy
}

Kilian Roßmann ${ }^{1,5}$, Kerem C. Akkaya ${ }^{2,5}$, Corentin Charbonnier ${ }^{1}$, Jenny Eichhorst ${ }^{2}$, Ben Jones ${ }^{3}$, Martin Lehmann",*, and Johannes Broichhagen ${ }^{1,4, *}$

${ }^{1}$ Department of Chemical Biology, Forschungsinstitut für Molekulare Pharmakologie, Berlin, Germany.

2 Department of Pharmacology and Cell Biology, Forschungsinstitut für Molekulare Pharmakologie, Berlin, Germany.

${ }^{3}$ Section of Endocrinology and Investigative Medicine, Imperial College London, London W12 0NN, United Kingdom.

4 Department of Chemical Biology, Max Planck Institute for Medical Research, Heidelberg, Germany.

5 These authors contributed equally.

* To whom correspondence should be addressed:

mlehmann@fmp-berlin.de and broichhagen@fmp-berlin.de 


\begin{abstract}
Rhodamine molecules are setting benchmarks in fluorescence microscopy. Herein, we report the deuterium (d12) congeners of tetramethyl(silicon)rhodamine, obtained by isotopic labelling of the four methyl groups, which improves photophysical (i.e. brightness, lifetimes) and chemical (i.e. bleaching) properties. We explore this finding for SNAP- and Halo-tag labelling, and highlight enhanced properties in several applications, such as Förster resonance energy transfer, fluorescence activated cell sorting, fluorescence lifetime microscopy and stimulated emission depletion nanoscopy. We envision deuteration as a generalizable concept to improve existing and develop new Chemical Biology Probes.
\end{abstract}

\title{
MAIN TEXT
}

Fluorescence microscopy is the technique of choice in modern biomedical research to elucidate structures or to interrogate function. Efforts to improve performances resulted in the development of super-resolution microscopy (nanoscopy), which is defined to obtain resolution higher than the diffraction limit described by Abbe's law. ${ }^{[1]}$ While optics and instruments have been advanced constantly, the elaboration of synthetic molecular dyes has driven the field to the current state-ofart. ${ }^{[2]}$ Given their small size, and the possibility to target them to cellular organelles (by means of substitution patterns) or proteins (by means of self-labelling tags, e.g. SNAP/Halo-tag fusions) make them an attractive choice for the visualization and interrogation of biomolecular function. ${ }^{[3]}$ Several chemical scaffolds, for instance nitrobenzodioxazoles $(\mathrm{NBDs})^{[4]}$ and coumarins ${ }^{[5]}$, remain interesting synthetic targets, yet xanthene dyes, which include tetramethylrhodamine (TMR) and silicon rhodamine ( $\mathrm{SiR})$, experienced a renaissance in terms of novel modifications to tune and boost important parameters, such as color, brightness, lifetime and reactivity. ${ }^{[6-10]}$ However, and best to our knowledge, isotopic labelling has not been among this repertoire.

We fill this gap by designing, synthesizing, and testing tetramethylrhodamines, in which each $\mathrm{CH}_{3}$ group is replaced by $\mathrm{CD}_{3}$, and furthermore equipped with a bioorthogonal handle for SNAP- or Halo-tag labelling of fused proteins (Fig. 1A, Scheme S1). Comparing deuterated and conventional rhodamines, we intuitively find that some photophysical properties remained unchanged, such as maximal excitation and emission wavelengths $\left(\lambda_{\mathrm{Ex} / \mathrm{Em}}(\mathrm{TMR}(-\mathrm{d} 12)) \sim 549 / 576\right.$ $\mathrm{nm} ; \lambda_{\mathrm{Ex} / \mathrm{Em}}(\operatorname{SiR}(-\mathrm{d} 12)) \sim 650 / 670 \mathrm{~nm}$ ) (Fig. 1A, B), while others do change dramatically, such as extinction coefficients $\left(\varepsilon(\mathrm{TMR})=78,000^{[6]} v s . \varepsilon(\mathrm{TMR}-\mathrm{d} 12)=83,000 \mathrm{M}^{-1} \mathrm{~cm}^{-1} ; \varepsilon(\mathrm{SiR})=141,000^{[6]}\right.$ 
vs. $\left.\varepsilon(\operatorname{SiR}-\mathrm{d} 12)=139,000 \mathrm{M}^{-1} \mathrm{~cm}^{-1}\right)$ and quantum yields $(\Phi(\mathrm{TMR})=0.43$ vs. $\Phi(\mathrm{TMR}-\mathrm{d} 12)=0.55$; $\left.\Phi(\mathrm{SiR})=0.41^{[11]} v s . \mathrm{SiR}-\mathrm{d} 12=0.57\right)$, leading to augmented brightness $(\varepsilon \times \Phi: \mathrm{TMR}=34$ vs. TMR-d12 = 46; $\mathrm{SiR}=0.58$ vs. SiR-d12 = 0.79) (Fig. 1A). Chemical fusion to $O^{6}$-benzylguanine or a chloroalkane linker yields labelling substrates for the SNAP- and Halo-tag, respectively. The in vitro labelling of SNAP with BG-TMR-d12 and BG-SiR-d12 could be traced by an increase in fluorescence polarization to determine labelling kinetics, which do not differ between hydrogenated and deuterated substrates $\left(\mathrm{t}_{1 / 2}(\mathrm{TMR})=13.3\right.$ vs. $\mathrm{t}_{1 / 2}(\mathrm{TMR}-\mathrm{d} 12)=12.2 \mathrm{~s} ; \mathrm{t}_{1 / 2}(\operatorname{SiR})=$ 23.5 vs. $\left.\mathrm{t}_{1 / 2}(\mathrm{SiR}-\mathrm{d} 12)=29.4 \mathrm{~s}\right)$ (Fig. 1A, C). By labelling a SNAP-Halo construct with HaloTMR/BG-SiR and BG-TMR/Halo-SiR, the improved photophysical properties lead to an increased efficiency in Förster resonance energy transfer (FRET) by $2 \%$ and $8 \%$, respectively, for the d12 variants (Fig. 1D). These results highlight that even subtle chemical changes can have pronounced effects on spectroscopic properties, exploring the chemical space in a new direction.

We next turned to cellular labelling and imaging and aimed to determine the behavior of our dyes on targets that are mainly expressed on the membrane, as well as targets that are found intracellularly, both regions that have been addressed for SNAP-tag labelling. ${ }^{[3]}$ First, we employed CHO-K1 cells stably expressing SNAP-tagged glucagon-like peptide 1 receptor (SNAPGLP1R:CHO-K1 ${ }^{[12]}$, a cell line intensely used to study the physiology of this blockbuster class B $\mathrm{G}$ protein-coupled receptor (GPCR) involved in glucose homeostasis and targeted in diabetes treatment $^{[13,14]}$, as a benchmark for $\mathrm{d} 12$ performances. As such, cells were labelled with $1 \mu \mathrm{M}$ BGTMR/SiR(-d12) for $30 \mathrm{~min}$, before washing and live imaging by confocal microscopy, which revealed staining of SNAP-GLP1R with all deuterated and parental dyes tested (Fig. 2A). Having established labelling on the outer plasma membrane, we secondly investigated intracellular staining in HeLa cells that stably express SNAP-tagged Cox8A (SNAP-Cox8A:HeLa), located in the inner mitochondrial membrane (Fig. 2B), which has been used to study mitochondrial ultrastructure in live cells. ${ }^{[15,16]}$ As for SNAP-GLP1R, we observed clean labelling with all dyes, and for both colors with an observable increase in brightness for the $\mathrm{d} 12$ derivatives. With this enhanced performance in microscopy, we wanted to quantify brightness by fluorescence activated cell sorting (FACS) to obtain robust values over large sample sizes. Accordingly, we labelled SNAP-GLP1R:CHO-K1 and SNAP-Cox8A:HeLa cells with both, BG-TMR(-d12) and BG-SiR(d12) to compare red and far-red color intensities by subsequent sorting (Fig. 2C). Histograms of SNAP-GLP1R:CHO-K1 cells labelled showed a right-shift in fluorescence intensity when dyes 
were deuterated (Fig. 2C, left). In contrast, SNAP-Cox8A:HeLa cells were labelled more homogeneously with a pronounced shift to higher intensities for SiR-d12 compared to its nondeuterated congener (Fig. 2C, right), while TMR(d12) only displayed a subtle change. By normalizing intensities and comparison, we calculate higher mean intensities for our deuterated dye versions (Fig. 2D). While no large increase was observed in SNAP-Cox8A:HeLa cells for TMR-d12 (2\%), mean intensity was markedly increased in SNAP-GLP1R:CHO-K1 cells (28\%). Furthermore, SiR-d12 outperformed SiR on SNAP-GLP1R and SNAP-Cox8A with an intensity increase of $43 \%$ and $50 \%$, respectively, which compares well with in vitro increase in brightness $(35 \%$ and $\mathrm{XX} \%)$. These results demonstrate that rhodamines with $\mathrm{CD}_{3}$ groups on the amines are not only the applicable to live cells but even outperform non-deuterated fluorophores, which is in line with our in vitro data of the unbound dyes.

With these encouraging results, we decided to test our deuterated probes in fluorescent lifetime confocal (FLIM) and stimulated emission by depletion (STED) microscopy, both stateof-the-art imaging techniques to reveal cellular dynamics and structures. Intriguingly, fluorescent lifetimes where higher for $\mathrm{d} 12$ congeners than their parent counterparts $(\tau(\mathrm{TMR})=2.3 v s . \tau(\mathrm{TMR}-$ $\mathrm{d} 12)=2.6 \mathrm{~ns} ; \tau(\mathrm{SiR})=2.9$ vs. $\tau(\mathrm{SiR}-\mathrm{d} 12)=3.5 \mathrm{~ns})($ Fig. 3A). However, TMR-d12 was not as susceptible to bleaching as TMR, while SiR and SiR-d12 exhibited similar trends of not being prone to fast bleaching in this setup (Fig. 3B). Lastly, and with SiR being one of the most successful far-red dyes for nanoscopy, we investigated super-resolution images acquired in live SNAP-Cox8A:HeLa cells. After incubation with $1 \mu \mathrm{M}$ BG-SiR or BG-SiR-d12, we recorded images of mitochondrial cristae under the same imaging conditions (Fig. 3C). While both dyes displayed labelling, SiR-d12 was able to resolve cristae sharper with less background, demonstrating its power in nanoscopy.

In our study, we synthesized and tested deuterated fluorophores. We install labelling moieties for SNAP- and Halo-tag conjugation and apply them in different experimental setups, i.e. in vitro FRET, and in cellulo confocal microscopy, FACS, FLIM and STED. We found that our fluorescent xanthene-based dyes TMR-d12 and SiR-d12 are improved by hydrogen-deuterium exchange on their methyl groups, enhancing photophysical parameters, such as brightness and lifetime, while reducing critical chemical parameters, such as bleaching. While the exact reason for this is unknown, we argue the following: i) affecting the rotation around the aromatic carbonnitrogen bond (in our case due to higher mass of the $\mathrm{CD}_{3}$ groups) has marked effects on fluorescent 
properties $^{[17]}$, which could suppress non-radiative decays and in turn enhances quantum yield and lifetime ${ }^{[6]}$; and ii) a lower zero-point energy of the $\mathrm{C}-\mathrm{D}$ vs. $\mathrm{C}-\mathrm{H}$ bond results in slower reaction kinetics, as an higher energy barrier has to be overcome ${ }^{[18]}$, and this could reduce bleaching through for example generated reactive oxygen species. While these explanations need more experimentation, ideally in combination with in silico calculations, we showcase novel deuterated dyes that outperform their parent molecules in multiple experiments, ranging from in vitro FRET, to live cellular labelling and sorting, in lifetime and super-resolution microscopy. We anticipate this concept i) to be generalizable to more xanthenes (e.g. fluoresceins, rhodols, SNARFs, and quenchers like QSYs), and other dye scaffolds (e.g. coumarins, cyanines, BODIPYs, EDANS, NBDs, Hoechst) including quenchers at any $\mathrm{C}-\mathrm{H}$ bond positions for improving and fine-tuning spectroscopic properties; ii) to be further explored with other isotopes, such as ${ }^{13} \mathrm{C},{ }^{15} \mathrm{~N}$ or even radioactive ${ }^{3} \mathrm{H}$; iii) to be used in different labelling approaches, such as the attachment to sulfonated BG (SBG) scaffolds allowing the separation of SNAP-tagged receptor pools ${ }^{[19]}$, to biomolecule targeting probes ${ }^{[20-22]}$, to "click chemistry" reagents (e.g. cyclopropenes, cyclooctenes) ${ }^{[23]}$ or to photoswitchable ligands ${ }^{[24,25]}$ including black hole quenchers (BHQs), and iv) to serve as multimodal dyes for confocal fluorescence and Raman microscopy ${ }^{[26]}$. These efforts are of ongoing interest in our laboratories. 


\section{METHODS}

\section{Synthesis}

Chemical synthesis (Supporting Scheme 1) and characterization of compounds is outlined in the Supporting Information. Purity of all dyes was determined to be of $>95 \%$ by UPLC-UV/Vis traces at $254 \mathrm{~nm}$ and dye specific $\lambda_{\max }$ that were recorded on a Waters H-class instrument equipped with a quaternary solvent manager, a Waters autosampler, a Waters TUV detector and a Waters Acquity QDa detector with an Acquity UPLC BEH C18 $1.7 \mu \mathrm{m}, 2.1$ x 50 mm RP column (Waters Corp., USA).

\section{Extinction coefficients, Excitation and Emission profiles and quantum yield.}

To assess photophysical parameters, ${ }^{1} \mathrm{H}$ NMR spectra of TMR(d12) and $\mathrm{SiR}(\mathrm{d} 12)$ were recorded with an internal standard (trimethoxybenzene, Aldrich \#74588-1g, standard for qNMR) to determine concentrations. The same solutions were diluted 1:1000 in activity buffer (containing in mM: $\mathrm{NaCl} 50$, HEPES 50, pH 7.3) or EtOH + 1\% TFA, and absorbance spectra were acquired on a NanodropOne 2000C. Extinction coefficients of d12 dyes were then calculated referenced to literature values of their non-deuterated parental molecules according to equation 1 .

$$
\varepsilon_{\mathrm{d} 12}=\varepsilon_{\mathrm{ref}} *\left(\left(\mathrm{Abs}_{\mathrm{d} 12} / \mathrm{Abs}_{\mathrm{ref}}\right) *\left(\mathrm{c}_{\mathrm{ref}} / \mathrm{c}_{\mathrm{d} 12}\right)\right)
$$

1:100 Dilutions in activity buffer were transferred into Greiner black flat bottom 96 well plates and excitation and emission profiles were recorded on a TECAN INFINITE M PLEX plate reader (TMR: $\lambda_{\mathrm{Ex}}=505 \pm 10 \mathrm{~nm} ; \lambda_{\mathrm{Em}}=550-800 \pm 20 \mathrm{~nm} ; 10$ flashes; $20 \mu$ s integration time; SiR: $\lambda_{\mathrm{Ex}}=$ $605 \pm 10 \mathrm{~nm} ; \lambda_{\mathrm{Em}}=640-800 \pm 20 \mathrm{~nm} ; 10$ flashes; $20 \mu$ s integration time). Absorbance values and integrated emission area (AUC) was used to calculate quantum yield (QY) according to equation 2 under the assumption that there is no change in refractive indices between solutions:

$$
\mathrm{QY}_{\mathrm{d} 12}=\mathrm{QY}_{\mathrm{ref}} *\left(\left(\mathrm{Abs}_{\mathrm{ref}} / \mathrm{Abs}_{\mathrm{d} 12}\right) *\left(\mathrm{AUC}_{\mathrm{d} 12} / \mathrm{AUC}_{\mathrm{ref}}\right)\right)
$$

Experiments were run in quadruplicate. Data normalization, integration and plotting was performed in GraphPad Prism 8.

\section{SNAP $f$ and SNAP-Halo expression and purification}

$\mathrm{SNAP}_{\mathrm{f}}$ was expressed and purified as described previously ${ }^{[16]}$ and complete amino acid sequences for constructs used can be found in the Supporting Information. SNAP-Halo with an N-terminal Strep-tag and C-terminal 10xHis-tag was cloned into a pET51b(+) expression vector for bacterial 
expression and complete amino acid sequences for constructs used can be found in the Supporting Information. For purification, SNAP-Halo was expressed in the E. coli strain BL21 pLysS. LB media contained ampicillin $(100 \mu \mathrm{g} / \mathrm{mL})$ for protein expression. A culture was grown at $37{ }^{\circ} \mathrm{C}$ until an $\mathrm{OD}_{600}$ of 0.6 was reached at which point cells were induced with IPTG $(0.5 \mathrm{mM})$. Protein constructs were expressed overnight at $16^{\circ} \mathrm{C}$. Cells were harvested by centrifugation and sonicated to produce cell lysates. The lysate was cleared by centrifugation and purified by Ni-NTA resin (Thermofisher) and Strep-Tactin II resin (IBA) according to the manufacturer's protocols. Purified protein samples were aliquoted in activity buffer (containing in $\mathrm{mM}$ : $\mathrm{NaCl}$ 50, HEPES 50, pH 7.3), flash frozen and stored at $-80{ }^{\circ} \mathrm{C}$.

\section{SNAP $_{\mathbf{f}}$ labelling kinetics}

Kinetic measurements were performed on a TECAN Spark Cyto and on a TECAN GENios Pro plate reader by means of fluorescence polarization. Stocks of $\operatorname{SNAP}_{\mathrm{f}}(400 \mathrm{nM})$ and substrates $(100$ $\mathrm{nM}$ ) were prepared in activity buffer (containing in $\mathrm{mM}$ : $\mathrm{NaCl} 50$, HEPES 50, $\mathrm{pH} 7.3$ ) with additional $10 \mu \mathrm{g} / \mathrm{mL}$ BSA. SNAP $\mathrm{f}$ and substrates were mixed $(100 \mu \mathrm{L}$ each) in a Greiner black flat bottom 96 well plate. Mixing was performed via a built-in injector on a TECAN GENios Pro or by manual pipetting on a TECAN Spark Cyto for TMR and SiR substrates, respectively. Fluorescence polarization reading was started immediately (TMR: $\lambda_{\mathrm{Ex}}=535 \pm 25 \mathrm{~nm} ; \lambda_{\mathrm{Em}}=590 \pm 35$ $\mathrm{nm} ; 10$ flashes; $40 \mu \mathrm{s}$ integration time; SiR: $\lambda_{\mathrm{Ex}}=605 \pm 20 \mathrm{~nm} ; \lambda_{\mathrm{Em}}=670 \pm 20 \mathrm{~nm} ; 10$ flashes; 40 $\mu \mathrm{s}$ integration time). Experiments were run in five repetitions and raw polarization values were one-phase decay fitted in GraphPad Prism 8.

\section{Protein labelling for FRET and full protein mass spectrometry}

For protein labelling, $1 \mu \mathrm{L}$ of the corresponding dye(s) (200 $\mu \mathrm{M}$ in DMSO) were diluted in $220 \mu \mathrm{L}$ of a $227 \mathrm{nM}$ solution of SNAP-Halo in activity buffer $(10 \mu \mathrm{g} / \mathrm{mL}$ BSA was added to controls where no SNAP-Halo protein was present). This resulted in a $\sim$-fold excess of labelling substrate and mixing was ensured by carefully pipetting the solution up and down. The reaction mixture was allowed to incubate at r.t. for $1 \mathrm{~h}$ before $20 \mu \mathrm{L}$ were removed for QToF MS analysis to ensure full labelling. The remaining solutions were subjected to spin column purification (Sartorius Vivaspin $50030 \mathrm{kDa}$ MWCO PES, \#VS0122) for three times by adding $500 \mu \mathrm{L}$ of activity buffer for each cycle. Finally, the solutions were reconstituted in activity buffer, of which $200 \mu \mathrm{L}$ were transferred 
into a Greiner black flat bottom 96 well plate and emission spectra were recorded on a TECAN INFINITE M PLEX (TMR: $\lambda_{\mathrm{Ex}}=510 \pm 10 \mathrm{~nm} ; \lambda_{\mathrm{Em}}=550-800 \pm 20 \mathrm{~nm} ; 25$ flashes; $20 \mu \mathrm{s}$ integration time; SiR: $\lambda_{\mathrm{Ex}}=610 \pm 10 \mathrm{~nm} ; \lambda_{\mathrm{Em}}=650-800 \pm 20 \mathrm{~nm} ; 10$ flashes; $20 \mu$ s integration time) to observe FRET. Donor or acceptor only labelled constructs, and donor plus acceptor with the addition of 10 $\mu \mathrm{g} / \mathrm{mL}$ BSA served as controls, and in these cases acceptor emission was not observed. FRET efficiency was calculated from the sum of maximal emission values derived from the raw spectra $\mathrm{I}_{\mathrm{TMR}}(572-584 \mathrm{~nm})$ and $\mathrm{I}_{\mathrm{SiR}}(660-672 \mathrm{~nm})$ according to equation 3:

(3) $\mathrm{E}_{\mathrm{FRET}}=\mathrm{I}_{\mathrm{SiR}} /\left(\mathrm{I}_{\mathrm{TMR}}+\mathrm{I}_{\mathrm{SiR}}\right)$

\section{Cell culture and FACS of SNAP-GLP1R:CHO-K1 and SNAP-Cox8A:HeLa cells}

CHO-K1 cells stably expressing the human SNAP-GLP1R (Cisbio) (SNAP-GLP1R:CHO-K1) were maintained in DMEM supplemented with $10 \% \mathrm{FCS}, 1 \%$ penicillin $/$ streptomycin, $500 \mu \mathrm{g} / \mathrm{mL}$ G418, $25 \mathrm{mM}$ HEPES, 1\% nonessential amino acids and 2\% L-glutamine. HeLa cells stably expressing SNAP-Cox8A ${ }^{[15]}$ (Cox8A-SNAP:HeLa) were maintained in DMEM supplemented with $10 \%$ FCS, $1 \%$ penicillin/streptomycin. Cells were incubated with $1 \mu \mathrm{M}$ of BG-conjugated dye for $30 \mathrm{~min}$ at $37{ }^{\circ} \mathrm{C}, 5 \% \mathrm{CO}_{2}$, washed with $\mathrm{PBS}$ w/o $\mathrm{Ca}^{2+}$ and $\mathrm{Mg}^{2+}$ and detached using Trypsin/EDTA. Detached cells were pelleted at $300 \mathrm{~g}$ at $4{ }^{\circ} \mathrm{C}$, resuspended in DPBS w/o $\mathrm{Ca}^{2+} / \mathrm{Mg}^{2+}$ and kept on ice until fluorescence activated cell sorting (FACS). FACS was performed in LSR-Fortessa (BD Bioscience) on cells gated by SSC/FSC using $561 \mathrm{~nm}$ excitation and BP 586/18 (561-586/18 BD channel) for TMR(d12) or $640 \mathrm{~nm}$ excitation and BP 670/30 (640-670/30 BD channel) for $\operatorname{SiR}(\mathrm{d} 12)$. Mean fluorescence intensity of $>10,000$ cells was analyzed and measured using Flow Jo (BD Bioscience).

\section{FLIM, Confocal and super-resolution microscopy}

Live cell fluorescence lifetime and confocal microscopy on SNAP-GLP1R:CHO-K1 was performed using a Leica SP8 with FALCON (Leica Microsystems) equipped with a pulsed whitelight excitation laser (80 MHz repetition rate (NKT Photonics)), a 100x objective (HC PL APO CS2 $100 \times / 1.40$ NA oil), a temperature controlled chamber and operated by LAS X. TMR(d12) were excited using $\lambda=561 \mathrm{~nm}$ and emission signals were captured at $\lambda=576-670 \mathrm{~nm}$. SiR(d12) were excited using $\lambda=640 \mathrm{~nm}$ and emission signals were captured at $\lambda=655-748 \mathrm{~nm}$. The emission signals were collected a time gated Hybrid detector (0.5-6 ns). FLIM images of sufficient 
signal were acquired without gating on Hybrid detectors within $512 \times 512$ pxl of $114 \mathrm{~nm} / \mathrm{pxl}$ with 10 repetitions. Fluorescence lifetime decay curves from selected regions with clear plasma membrane staining were fitted with two exponential functions and the mean amplitude weighted lifetime is reported for each region.

Confocal fluorescence microscopy for photobleaching experiments SNAP-GLP1R were performed on living cells in PBS at room temperature on LSM710 or LSM780 (Carl Zeiss) operated by Zen Black Software using a 63x (1.40 NA oil) objective. TMR(d12) were excited using $\lambda=561 \mathrm{~nm}$ and emission signals were captured at $\lambda=564-712 \mathrm{~nm}$. SiR(d12) were excited using $\lambda=633 \mathrm{~nm}$ and emission signals were captured at $\lambda=637-740 \mathrm{~nm}$. Emission signal were collected on 34 channel spectral detector (QUASAR, Zeiss), a typical gain of 750 over a scan area of $512 \times 512 \mathrm{pxl}$.

Confocal and STED microscopy experiments on living Cox8A-SNAP:HelA cells were performed in Life cell imaging buffer (Gibco) at $37^{\circ} \mathrm{C}$ using a 100x (1.45 NA lambda oil) objective on a Nikon TiEclipse operated by Micromanager and equipped with a STEDyCON (Abberior Instruments) with 405/488/561/640 excitation and a $775 \mathrm{~nm}$ depletion laser. TMR(d12) were excited using $\lambda=561 \mathrm{~nm}$ and emission signals were captured at $\lambda=580-630 \mathrm{~nm}$. SiR(d12) were excited using $\lambda=640 \mathrm{~nm}$ and emission was collected at $\lambda=650-700 \mathrm{~nm}$. Both Emission signals were collected by a time gated APD (0.5-8 ns) with $8 x$ signal accumulation and $100 \mathrm{~nm}$ pixel size for confocal images. STED images for SiR(d12) were collected with $41 \% 775 \mathrm{~nm}$ depletion laser power and $15 \mathrm{~nm}$ pixel size.

\section{Acknowledgements}

BJ is supported by the Medical Research Council, European Association for the Study of Diabetes, Society for Endocrinology, British Society for Neuroendocrinology and the NIHR Imperial Biomedical Research Centre. We thank Andrea Bergner and Julien Hiblot for providing purified $\mathrm{SNAP}_{\mathrm{f}}$ protein, Bettina Mathes and Sarah Mikami for synthetic assistance, Cornelia Ulrich and Sebastian Fabritz for mass spectrometry, Kai Johnsson for providing SNAP plasmids, chemical precursors and constant support (all MPIMR). We are grateful to Martina Leidert and Anne Diehl for protein expression and purification, Peter Schmieder for NMR, Ramona Birke and the Hackenberger Laboratory for support (all FMP). 


\section{Author Contributions}

JB conceived and supervised the study. JB designed, synthesized and characterized chemical compounds. KR, CC and JB performed in vitro measurements. KCA, JE and ML performed cell culture, cell sorting and microscopy. BJ provided reagents. JB wrote the manuscript with input from all authors.

\section{ORCIDs}

$\begin{array}{ll}\text { Kilian Rossmann } & 0000-0003-4965-9669 \\ \text { Kerem C. Akkaya } & 0000-0002-6214-2229 \\ \text { Corentin Charbonnier } & 0000-0001-5782-7058 \\ \text { Jenny Eichhorst } & \text { N/A } \\ \text { Ben Jones } & 0000-0003-0461-2584 \\ \text { Martin Lehmann } & 0000-0002-8370-6353 \\ \text { Johannes Broichhagen } & 0000-0003-3084-6595\end{array}$

\section{Competing Interests}

The authors declare no competing interests.

\section{References}

[1] S. J. Sahl, S. W. Hell, S. Jakobs, Nature Reviews Molecular Cell Biology 2017, 18, 685-701.

[2] S. W. Hell, Angewandte Chemie International Edition 2015, 54, 8054-8066.

[3] L. Xue, I. A. Karpenko, J. Hiblot, K. Johnsson, Nature Chemical Biology 2015, 11, 917-23.

[4] S. Benson, A. Fernandez, N. D. Barth, F. de Moliner, M. H. Horrocks, C. S. Herrington, J. L. Abad, A. Delgado, L. Kelly, Z. Chang, Y. Feng, M. Nishiura, Y. Hori, K. Kikuchi, M. Vendrell, Angewandte Chemie International Edition 2019, 58, 6911-6915.

[5] S. S. Matikonda, J. Ivanic, M. Gomez, G. Hammersley, M. J. Schnermann, Chemical Science 2020, 11, 7302-7307.

[6] J. B. Grimm, B. P. English, J. Chen, J. P. Slaughter, Z. Zhang, A. Revyakin, R. Patel, J. J. Macklin, D. Normanno, R. H. Singer, T. Lionnet, L. D. Lavis, Nature Methods 2015, 12, 244-250.

[7] L. Wang, M. Tran, E. D’Este, J. Roberti, B. Koch, L. Xue, K. Johnsson, Nature Chemistry 2020, 12, 165-172.

[8] A. N. Butkevich, G. Y. Mitronova, S. C. Sidenstein, J. L. Klocke, D. Kamin, D. N. H. Meineke, E. D’Este, P.-T. Kraemer, J. G. Danzl, V. N. Belov, S. W. Hell, Angewandte Chemie International Edition 2016, 55, 3290-3294. 
[9] A. Wieczorek, P. Werther, J. Euchner, R. Wombacher, Chemical Science. 2017, 8, 15061510 .

[10] Q. Zheng, A. X. Ayala, I. Chung, A. V. Weigel, A. Ranjan, N. Falco, J. B. Grimm, A. N. Tkachuk, C. Wu, J. Lippincott-Schwartz, R. H. Singer, L. D. Lavis, ACS Central Science 2019, 5, 1602-1613.

[11] G. Lukinavičius, K. Umezawa, N. Olivier, A. Honigmann, G. Yang, T. Plass, V. Mueller, L. Reymond, I. R. Corrêa Jr, Z.-G. Luo, C. Schultz, E. A. Lemke, P. Heppenstall, C.

Eggeling, S. Manley, K. Johnsson, Nature Chemistry 2013, 5, 132-139.

[12] T. Buenaventura, S. Bitsi, W. E. Laughlin, T. Burgoyne, Z. Lyu, A. I. Oqua, H. Norman, E. R. McGlone, A. S. Klymchenko, I. R. C. Jr, A. Walker, A. Inoue, A. Hanyaloglu, J. Grimes, Z. Koszegi, D. Calebiro, G. A. Rutter, S. R. Bloom, B. Jones, A. Tomas, PLOS Biology 2019, 17, e3000097.

[13] Z. Fang, S. Chen, P. Pickford, J. Broichhagen, D. J. Hodson, J. Ivan R. Corrêa, S. Kumar, F. Görlitz, C. Dunsby, P. M. W. French, G. A. Rutter, T. Tan, S. R. Bloom, A. Tomas, B. Jones, ACS Pharmacology \& Translational Science 2020, DOI 10.1021/acsptsci.0c00022.

[14] P. Pickford, M. Lucey, Z. Fang, S. Bitsi, J. B. de la Serna, J. Broichhagen, D. J. Hodson, J. Minnion, G. A. Rutter, S. R. Bloom, A. Tomas, B. Jones, British Journal of Pharmacology 2020, ahead of print, DOI 10.1111/bph.15134.

[15] T. Stephan, A. Roesch, D. Riedel, S. Jakobs, Scientific Reports 2019, 9, 12419.

[16] T. Stephan, C. Brüser, M. Deckers, A. M. Steyer, F. Balzarotti, M. Barbot, T. S. Behr, G. Heim, W. Hübner, P. Ilgen, F. Lange, D. Pacheu-Grau, J. K. Pape, S. Stoldt, T. Huser, S. W. Hell, W. Möbius, P. Rehling, D. Riedel, S. Jakobs, The EMBO Journal 2020, 39, e104105.

[17] J. V. Jun, D. M. Chenoweth, E. J. Petersson, Organic \& Biomolecular Chemistry 2020, $18,5747-5763$.

[18] P. W. Atkins, Physical Chemistry., WHFreeman And Co, New York, 2010.

[19] P. Poc, V. A. Gutzeit, J. Ast, J. Lee, B. J. Jones, E. D’Este, B. Mathes, M. Lehmann, D. J. Hodson, J. Levitz, J. Broichhagen, Chemical Science 2020, 11, 7871-7883.

[20] S. Karch, J. Broichhagen, J. Schneider, D. Böning, S. Hartmann, B. Schmid, P. Tripal, R. Palmisano, C. Alzheimer, K. Johnsson, T. Huth, Journal of Medicinal Chemistry 2018, 61, 6121-6139.

[21] J. Ast, A. Arvaniti, N. H. F. Fine, D. Nasteska, F. B. Ashford, Z. Stamataki, Z. Koszegi, A. Bacon, B. J. Jones, M. A. Lucey, S. Sasaki, D. I. Brierley, B. Hastoy, A. Tomas, G. D’Agostino, F. Reimann, F. C. Lynn, C. A. Reissaus, A. K. Linnemann, E. D’Este, D. Calebiro, S. Trapp, K. Johnsson, T. Podewin, J. Broichhagen, D. J. Hodson, Nature Communications 2020, 11, 467.

[22] G. Lukinavičius, L. Reymond, E. D’Este, A. Masharina, F. Göttfert, H. Ta, A. Güther, M. Fournier, S. Rizzo, H. Waldmann, C. Blaukopf, C. Sommer, D. W. Gerlich, H.-D. Arndt, S. W. Hell, K. Johnsson, Nature Methods 2014, 11, 731-733.

[23] B. L. Oliveira, Z. Guo, G. J. L. Bernardes, Chemical Society Reviews 2017, 46, 48954950.

[24] K. Hüll, J. Morstein, D. Trauner, Chemical Reviews 2018, 118, 10710-10747.

[25] A. Acosta-Ruiz, V. A. Gutzeit, M. J. Skelly, S. Meadows, J. Lee, P. Parekh, A. G. Orr, C. Liston, K. E. Pleil, J. Broichhagen, J. Levitz, Neuron 2020, 105, 446-463.

[26] G. A. Matanfack, J. Rüger, C. Stiebing, M. Schmitt, J. Popp, Journal of Biophotonics 2020, ahead of print, e202000129. 


\section{Figures}

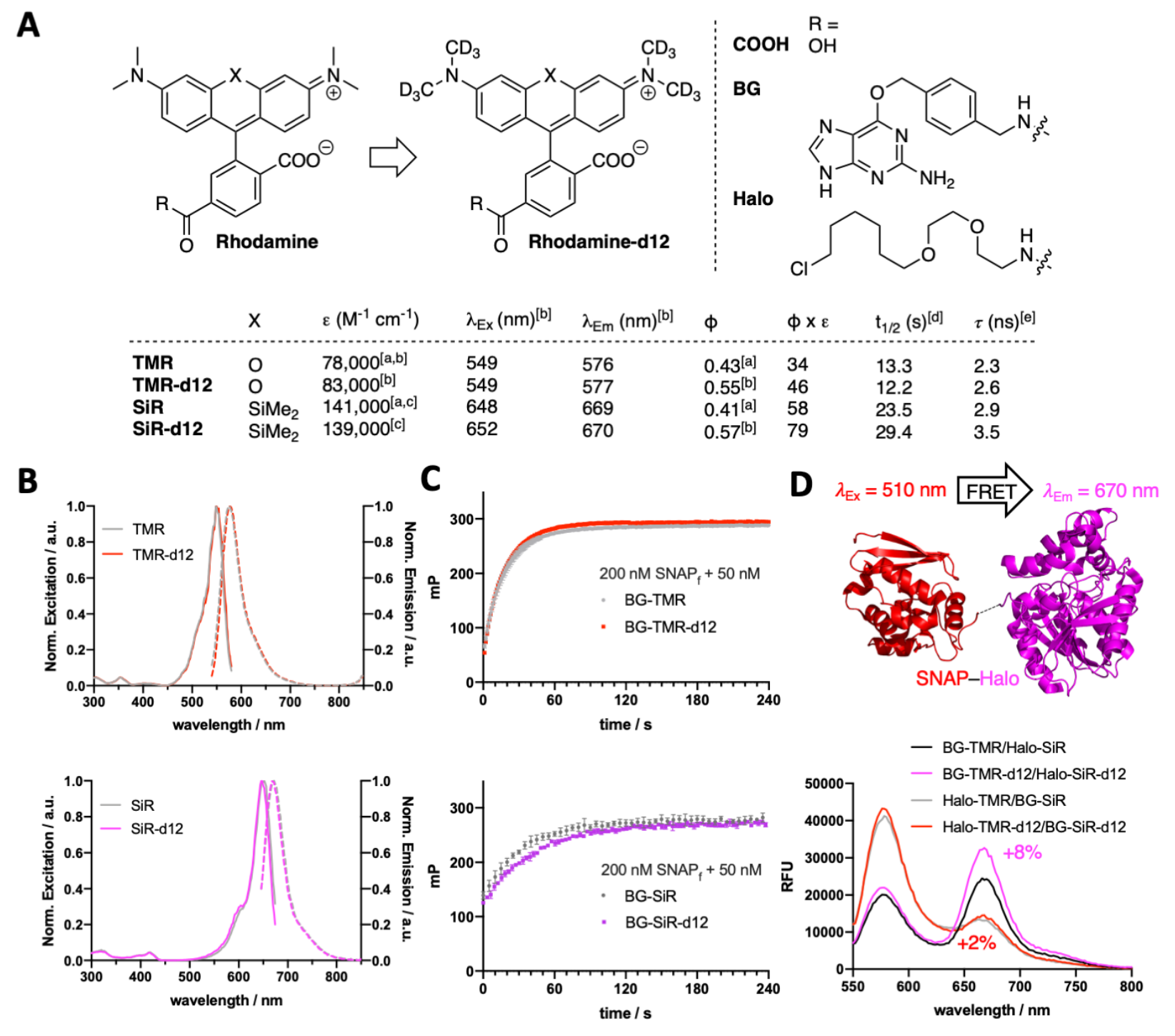

Figure 1. Deuteration improves rhodamine brightness. A) The isotopic H/D exchange on the four methyl groups leads to d12 variants of TMR $(X=O)$ and $\operatorname{SiR}\left(X=\operatorname{SiMe}_{2}\right)$. Derivatization on the 6-carboxylate allows synthesis of BG- and Halo-congeners for SNAP- and Halo-tag labelling, respectively. B) Excitation and emission spectra of TMR(-d12) (top) and SiR(-d12) (bottom). C) Fluorescence polarization (mP) assay of BG-TMR(-d12) (top) and BG-SiR(-d12) (bottom) when incubated with $\mathrm{SNAP}_{\mathrm{f}}$ to determine labelling kinetics. D) In vitro FRET assay of a BG-TMR(d12) and Halo-SiR(-d12) labelled SNAP-Halo protein shows an increase of acceptor/donor emission ratio. ${ }^{[\mathrm{a}]}$ reference values; ${ }^{[\mathrm{b}]}$ in activity buffer; ${ }^{[\mathrm{c}]}$ in $\mathrm{EtOH}+1 \% \mathrm{TFA}$; ${ }^{[\mathrm{d}]} \mathrm{R}=\mathrm{BG}$ and $\mathrm{SNAP}_{\mathrm{f}}$ in vitro in activity buffer; ${ }^{[\mathrm{e}]} \mathrm{SNAP}$ bound in cells. 

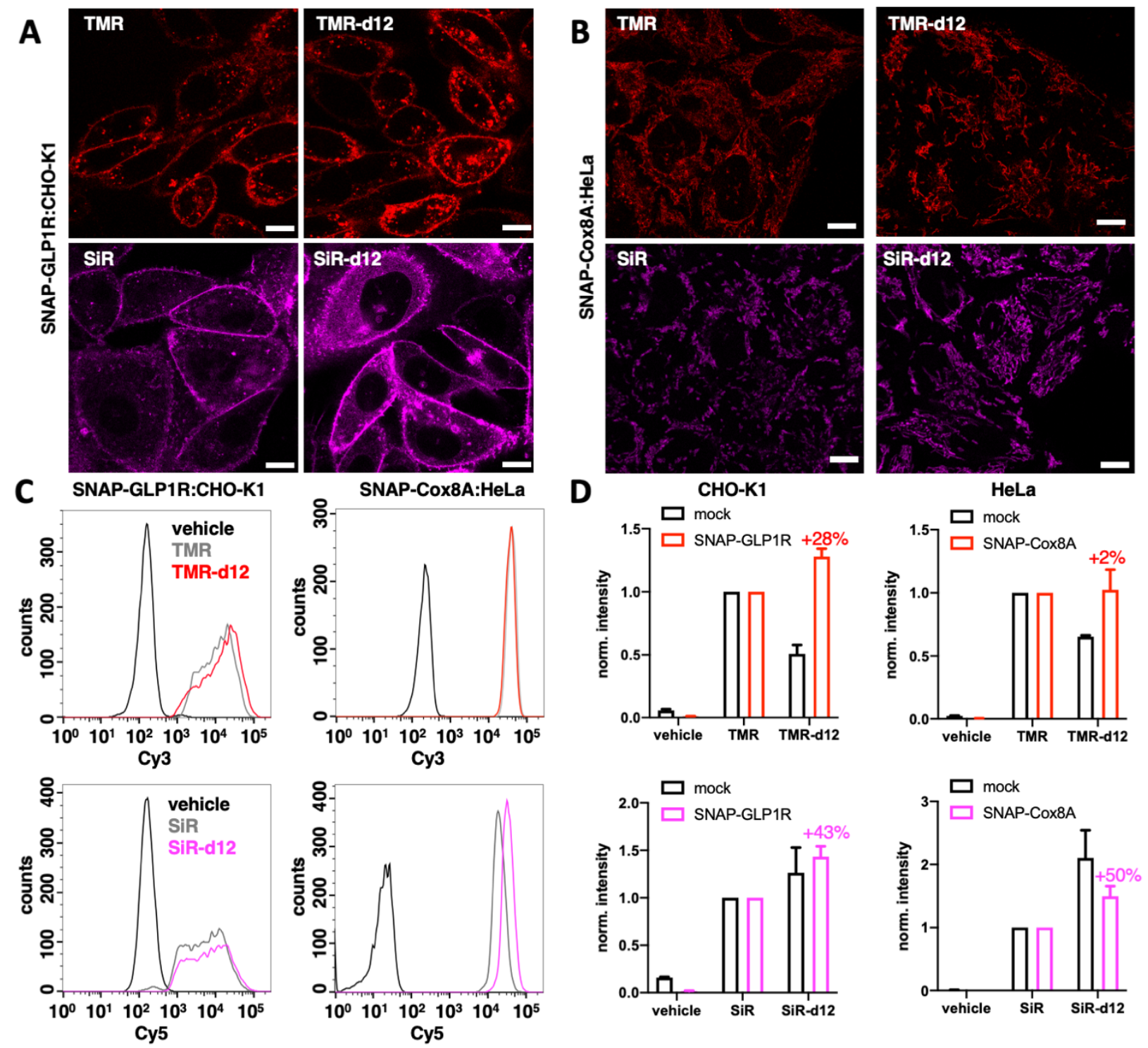

Figure 2. Microscopy and cell sorting reveal increased intensities of rhodamines. A) BGTMR(-d12) (top) and BG-SiR(-d12) (bottom) label stable SNAP-GLP1R expressing CHO-K1 cells. Scale bar $=10 \mu \mathrm{m}$. B) BG-TMR(-d12) (top) and BG-SiR(-d12) (bottom) label stable SNAPCox8A expressing HeLa cells. Scale bar $=10 \mu \mathrm{m}$. C) Raw data from cell sorting of BG-TMR(d12) (top) and BG-SiR(-d12) (bottom) labelled stable SNAP-GLP1R:CHO-K1 and SNAPCox8A:HeLa cells from A and B. D) As for C, normalized results including mock cells. $n=3$ independent experiments. Mean \pm SD. 
A
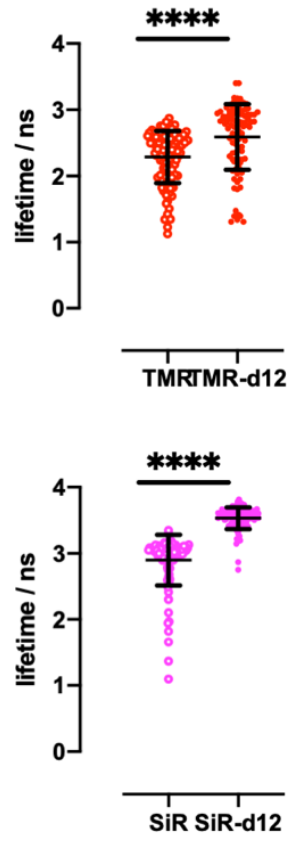

B
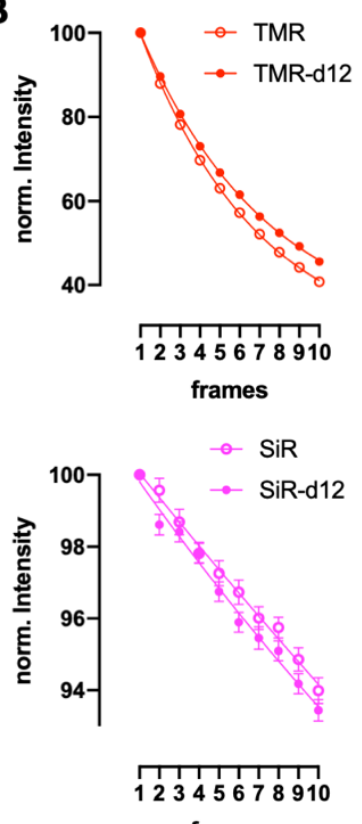

frames
C

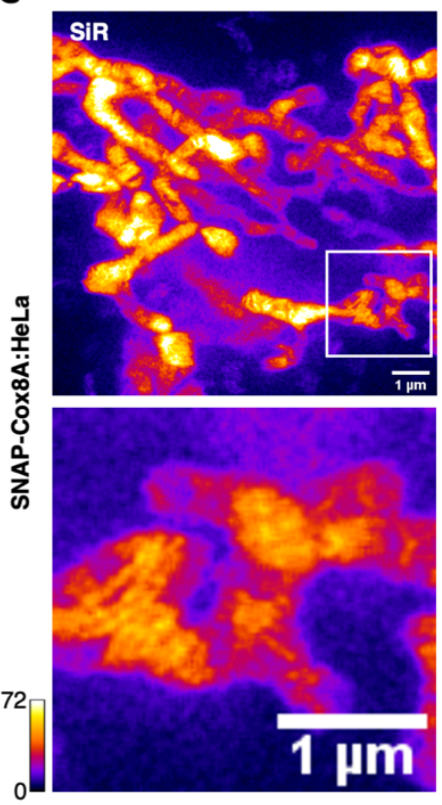

Figure 3. Deuteration increases rhodamine fluorescent lifetimes and shows improved performances and STED nanoscopy. A, B) Fluorescence lifetimes (A) and bleaching curves (B) of BG-TMR(-d12) (top) and BG-SiR(-d12) (bottom) labelled SNAP-GLP1R:CHO-K1 cells. Measurements from individual cells pooled form 2 independent experiments. Mean $\pm \mathrm{SD}$, **** indicates statistical significance (unpaired t-test, $\mathrm{p}<0.0001$ ). C) STED nanoscopy of BG-SiR (left) and BG-SiR-d12 (right) in live SNAP-Cox8A:HeLa cells resolving mitochondrial cristae under identical imaging conditions. Scale bar $=1 \mu \mathrm{m}$. Representative images of $>3$ independent experiments. 